\title{
AKTiveSA: A Technical Demonstrator System For Enhanced Situation Awareness
}

\author{
Paul R. Smart*, Alistair Russell, Nigel R. Shadbolt, M. C. Shraefel and Leslie A. Carr \\ School of Electronics and Computer Science, University of Southampton, Southampton SO17 1BJ, UK \\ *Corresponding author: ps02v@ecs.soton.ac.uk
}

\begin{abstract}
The issue of improved situation awareness is a key concern for military agencies, promising to deliver strategic advantages in a variety of conflict and non-conflict scenarios. Improved situation awareness can benefit operational effectiveness by facilitating the planning process, improving the quality and timeliness of decisions, and providing better feedback regarding the strategic consequences of military actions. In this paper, we aim to show how a combination of semantic technologies and user interface design initiatives can be used to improve situation awareness in a simulated humanitarian relief scenario. We describe the development of a technical demonstrator system, the AKTiveSA TDS, which integrates a variety of knowledge technologies and visualization components within the context of a unitary application framework. We also describe our approach to scenario development, knowledge acquisition, ontology engineering and system design. Some specific problems encountered during system development are discussed, e.g. the performance overheads associated with rules-based processing, and potential solution strategies for these problems are presented alongside a description of future development activities.
\end{abstract}

Keywords: situation awareness; semantic web; humanitarian relief; user interface design

Received 23 May 2007; revised 23 May 2007

\section{INTRODUCTION}

Situation awareness is a critical success factor in all military operations. Even when the operational context is not directly adversarial, as is the case in most humanitarian and peacekeeping operations, awareness of the temporal unfolding of events, the strategic displacement of military assets and the ability to anticipate the actions of other (sometimes competing) agencies, all serve to underpin the successful realization of operational objectives. The need for improved situation awareness is particularly important when one considers the increasingly sophisticated technological backdrop against which military operations are typically undertaken. The advent of network-enabled capabilities (NEC) and the growth of the internet as a medium for information dissemination, affords great opportunities for situation awareness, but it also presents some relatively new and distinct challenges. One challenge relates to the need to distinguish relevant information from background noise (the concern here is that highly relevant information may be swamped by less relevant information). Another relates to the need to carefully filter incoming information streams based on the knowledge and information requirements of different information consumers. Further worries relate to the rate of information dissemination in today's media-intensive environment. The concern here is that the dynamics of the situation picture may result in the differential prioritization of problem-solving goals. When goal switching is mandated by changing operational commitments then different subsets of information will need to be dynamically integrated or aggregated to support changing situation awareness concerns.

Some of the challenges to situation awareness in military contexts can, we argue, be addressed by a combination of semantic technologies and advanced modes of information visualization and user interaction. Semantic technologies, as developed in the context of the Semantic Web initiative [1] promise to provide a platform for information exchange and knowledge-oriented processing that meets many of the

The Computer Journal, Vol. 50 No. 6, 2007 
requirements of an enabling technology underpinning improved situation awareness. Ontologies provide a foundation for semantic interpretation and information fusion processes, while query languages, such as RDQL [2] and SPARQL [3], facilitate information search and retrieval in a manner that is specifically oriented to an agent's ongoing information needs and concerns. Semantically-enriched information also supports a variety of reasoning and inferential processes, and these have the potential to assist the user with respect to information triage and the execution of knowledgeintensive tasks. Above all we see semantic technologies as providing a mechanism for representing task-relevant information requirements, and for enabling rapid access to relevant information content in large-scale, distributed information environments. In essence, we see semantic technologies as providing the basis for semantic annotation schemes that can be used to facilitate adaptive modes of information aggregation in support of goal-relevant processing, a critical element of enhanced situation awareness [4].

User interfaces can also influence situation awareness. Not only is the user interface the primary medium for information transfer, and therefore the basis for initial stages of situation awareness (i.e. the level of perceptual processing, in Endlsey's [5] tripartite model), the features of the display device can also exert a significant effect on the depth to which situationrelevant information items are processed by human end-users [6]. In addition to these concerns, the user interface often serves as an important adjunct to temporally extended bouts of problem-solving activity: the user interface affords a number of opportunities for end-user interaction with a system and this permits a user to progressively restructure information content to better suit their idiosyncratic perceptual and cognitive capabilities. This strategy has much in common with the notion of 'epistemic action' in which a problemsolving agent initiates actions to restructure the problemsolving environment in a manner that simplifies subsequent problem-solving operations [7].

This paper describes a Technical Demonstrator System (TDS), developed as part of the Data and Information Fusion Defence Technology Centre (DIF DTC) Phase I AKTiveSA project at the University of Southampton. The TDS (hereafter referred to as the AKTiveSA TDS) attempts to highlight the role played by semantic and visualization technologies in enhancing situation awareness in a simulated humanitarian relief scenario. The description of system capabilities presented here extends earlier characterizations of a prototype system which was described by Smart et al. [8, 9].

\section{OPERATIONAL CONTEXT}

In order to provide a context for knowledge engineering and technology development, we surveyed a variety of operational contexts as a precursor to scenario development (Section 3). We selected humanitarian/disaster relief operations [10] primarily because they entail the exploitation of information resources that subtend the civil and military information domains. There is often a need in these operational contexts to integrate heterogeneous information content, much of which may be distributed in large-scale, open-access, information environments, such as the World Wide Web (WWW). This provides a suitable context for Semantic Web technologies because such technologies were developed to support information access and integration in precisely these types of information environment.

Another reason for the choice of operational context relates to the focus of current military engagements. Since the end of the Cold War period armed forces have been increasingly involved in humanitarian and disaster relief efforts. The United Nations High Commissioner for Refugees [11] has identified a number of reasons for this, including:

(i) Military factors. Humanitarian actions may be undertaken as part of an effort to secure the good will of the population within which a force is operating.

(ii) Institutional factors. Military forces may be tasked with peace support and relief operations because there is a need to foster the impression that they are active during periods of low threat.

(iii) Political factors. Military involvement may be encouraged as part of a public relations effort directed towards constituencies in the sending state.

The trend towards increasing military involvement in humanitarian affairs is generally seen as undesirable by humanitarian agencies, who regard the use of military assets as threatening to compromise the neutrality and impartiality of humanitarian actors. Nevertheless, the value of military forces in at least some aspects of humanitarian relief operations is widely accepted. For example, military agencies often have the capability to help secure an enabling environment in which humanitarian activities can take place in relative safety; they may also have the practical means to assist in the delivery of aid efforts, e.g. the rapid deployment of large numbers of personnel, equipment, logistics and supplies.

The nature of the cooperation between humanitarian and military agencies may assume a variety of forms. For example, the mandate of the United Nations (UN) peace keeping force in Afghanistan-the International Security Assistance Force $\left(\mathrm{ISAF}^{1}\right)$-includes support to UNsanctioned humanitarian and recovery efforts and it has a Civil-Military Cooperation group to perform this function. There are few limits on information exchange between ISAF and humanitarian agencies in Afghanistan; however, in general, the free exchange of information between military

\footnotetext{
${ }^{1}$ http://www.nato.int/ISAF/
}

The Computer Journal, Vol. 50 No. 6, 2007 
and humanitarian agencies is fraught with difficulties. The military cannot, for example, disclose classified information to humanitarian agencies, whereas humanitarian relief organizations are reluctant to share information that may look like 'intelligence' and thus threaten the security of their staff and operations in the field [12]. In general, the Inter-Agency Standing Committee identifies a number of types of information that may be shared between military and humanitarian agencies [13]. These include:

(i) Security information. Information relevant to the security situation in the area of operations.

(ii) Humanitarian locations. The coordinates of humanitarian staff and facilities inside the theatre-ofoperations.

(iii) Humanitarian activities. The plans and intentions of humanitarian actors (this aims at avoiding accidental strikes on humanitarian workers).

(iv) Mine action activities. Information relevant to mine action activities.

(v) Population movements. Information about major movements of civilians.

(vi) Military relief activities. Information about the relief efforts undertaken by military agencies.

(vii) Post-strike Information. Information on strike locations and explosive munitions used during military campaigns (this assists with the prioritization and planning of humanitarian relief and mine action/ UXO activities).

(viii) Transport infrastructure information. The integrity of roads and bridges and other transport routes.

(ix) Communication infrastructure. e.g. the best location for radio repeaters.

Such information serves as a useful point of departure for knowledge engineering (Section 4) because it indicates the types of information that may need to be processed in the context of humanitarian operations. ${ }^{2}$

\section{SCENARIO SPECIFICATION}

To further ground knowledge engineering and technical development activities, we developed a domain scenario within the scope of humanitarian/disaster relief operations [14]. The scenario is set in Afghanistan and features a number of humanitarian events; most notably an earthquake and a flood event, occurring against the backdrop of ongoing military conflict (Fig. 1).

In constructing the scenario we developed a storyboard of scenario events and used Google Earth, ${ }^{3}$ in conjunction with

\footnotetext{
${ }^{2}$ Mine action activities and post-strike information in fact constitute a focal point for knowledge modelling in the DIF DTC Phase II SEMIOTIKS cluster project, a successor to the AKTiveSA initiative.

${ }^{3}$ http://earth.google.com/
}

the Keyhole Markup Language $\left(\mathrm{KML}^{4}\right)$ to depict the situation picture at various points in the scenario timeline (Fig. 1). The representational capabilities of KML, in conjunction with the Google Earth client, proved invaluable in terms of rapidly creating a scenario storyboard within a complex, interactive 3-D environment. Furthermore, by exploiting the ability to incrementally modify KML data over a network, we were able to represent the time-variant dynamics of scenario elements (e.g. the movement of specific platforms), thereby providing a visually compelling and interactive walkthrough of the entire scenario storyboard. This technique was useful in terms of presenting the scenario to military stakeholders and also proved invaluable in terms of prototyping some of the later visualization and interactive capabilities of the AKTiveSA TDS.

\section{ONTOLOGY ENGINEERING}

The exploitation of domain knowledge in the current project, in conjunction with the use of semantic technologies, necessitated an analysis of the knowledge infrastructure for humanitarian and disaster relief operations. This was subsumed within a large-scale ontology engineering initiative that drew on state-of-the-art approaches to knowledge elicitation $[15,16]$, knowledge modelling [17] and knowledge representation [18].

\subsection{Ontology development}

Due to the complexity of the conceptual infrastructure of the problem domain (subsuming domains as diverse as military technology, humanitarian aid missions and meteorology) we required significant input from stakeholder communities, particularly UK military agencies, as the basis for initial knowledge capture and subsequent knowledge validation. Our approach to ontology development in the context of the current initiative assumed the form of a tripartite scheme in which the outputs of earlier phases were progressively refined and formalized in subsequent phases.

\subsubsection{Knowledge acquisition}

Our approach to knowledge acquisition was largely based around a number of knowledge elicitation sessions with military Subject Matter Experts (SMEs) at the Royal School of Artillery and the Land Warfare Centre. Knowledge capture assumed the form of direct observation of military planning activities and also entailed the use of interview techniques with subsequent protocol analysis of the interview transcripts. We also relied on a variety of knowledge acquisition techniques, such as laddered grids and process maps $[15,16]$ to organize and structure knowledge gleaned from a variety of

\footnotetext{
${ }^{4} \mathrm{KML}$ is an XML-compliant language used to represent geographic data within an Earth Browser, such as Google Earth, Google Maps, or Google Maps for mobile (http://earth.google.com/ $/ \mathrm{kml} /$ ).
} 
The AKTiveSA domain scenario is set in Afghanistan in the year 2002. US and UK forces have been deployed in Afghanistan to conduct strategic operations against the Taliban regime. Following a successful air campaign and land-based assault, the Taliban have been overthrown and major conflict operations have been concluded. Despite this, pockets of resistance remain in the southern and western regions of Afghanistan and the risk of both terrorist attacks against the local civilian population and assaults on coalition forces remains high. Thus far, the occurrence of such incidents has been largely restricted to the Kandahar Oruzgan, Zabul, Helmand and Farah provinces. US and UK ground forces have been deployed in-theatre both in the north and south of Afghanistan, and these units continue to undertake strategic operations against Taliban insurgents, largely within the provinces of Kandahar and Oruzgan These operations are backed up by Close Air Support (CAS) and air strikes against strategic targets in Kandahar. Ground forces have also been tasked with peace support, counter-terrorism and humanitarian relief activities in the occupied areas, mostly in the eastern provinces. In addition, the United Nations (UN) has deployed an International Security Assistance Force (ISAF) to assist with peace support operations.

The ongoing military conflict has created a refugee crisis in which many local residents have moved east to leave Afghanistan via the Pakistan border. Following the closure of this border a number of Internally Displaced Person (IDP) camps have been established in the east of the country and these continue to receive new inhabitants from both conflict zones and areas affected by military conflict. Humanitarian aid organizations are assisted in their duties by military units who provide both logistical support and security for aid distribution.

At 12:10 hours on 29th March 2002, seismometer readings indicate the occurrence of a large earthquake in the region of the Baghlan/Bamyan border region. Information about the earthquake is provided by the National Earthquake Information Center (NEIC), who indicate the magnitude and location (epicentre and hypocentre) of the Earthquake. This initial information is subsequently corroborated by other seismic monitoring centres. The location and magnitude of the earthquake indicate a medium intensity earthquake with associated structural damage and possible civilian casualties. Information from the Global Disaster Alert and Coordination System (GDACS) provides a characterization of the earthquake and also indicates its likely humanitarian impact.

In addition to the devastation caused by the earthquake in the immediate vicinity (mostly mediated by the collapse of buildings in local settlements) the magnitude of the earthquake is sufficient to cause the Band Sultan dam, located north of Ghazni, to collapse and cause massive inundation of the surrounding region. The resulting flood, exacerbated by the accumulation of water from melted snow in the dam reservoir, is responsible for the destruction of hundreds of houses, the death of at least 1000 people and the displacement of many more.
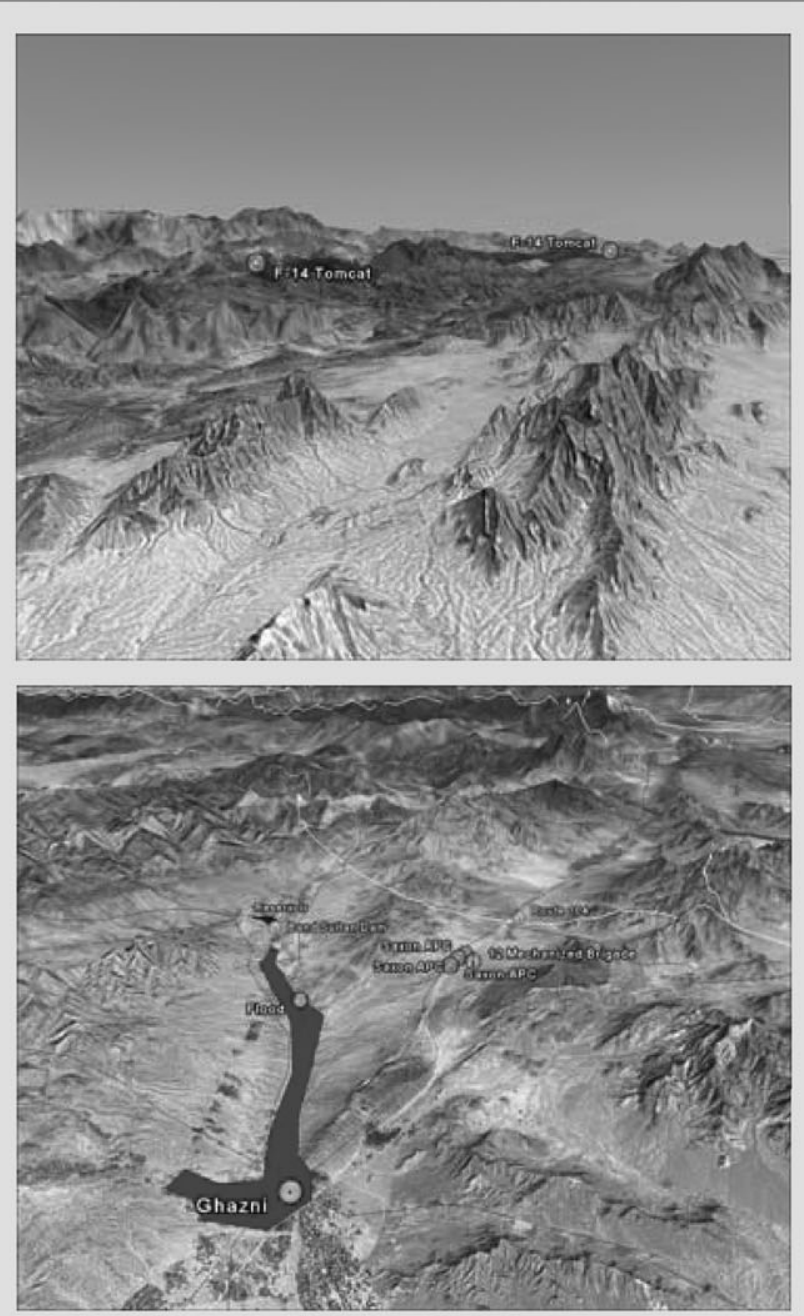

In response to the earthquake, US-led coalition and ISAF forces, in concert with humanitarian aid organizations, aim to provide humanitarian assistance to the afflicted region. The immediate humanitarian response assumes the form of food, shelter and medical aid provision. Military units are also tasked with search and rescue operations in the flooded region, the transport of aid supplies and the provision of security for humanitarian aid efforts. The relief effort is complicated by both the security situation and the damage caused to the local transport network by the flood water. A number of land routes in the region of the disaster have been blocked by rising flood water, which affects the overland transport of aid supplies. In addition, the security situation makes both land-based and airborne vehicles vulnerable to enemy fire, thereby making some transport routes hazardous and others impassable. As the crisis develops over the next few days there are reports of a number of outbreaks of water borne diseases and the possibility of an epidemic becomes a realistic possibility.

FIGURE 1. AKTiveSA domain scenario.

knowledge sources, including public domain resources (such as websites), military publications (e.g. doctrinal publications) and SME input.
Our knowledge acquisition efforts provided information about the types of events, entities and contingencies that were likely to be important for operationally-effective

The Computer Journal, Vol. 50 No. 6, 2007 
decision-making in humanitarian assistance missions. Example focus areas for knowledge acquisition included:

(i) information about the extent of involvement of military agencies in humanitarian assistance missions (particularly in situations where humanitarian aid is provided against a backdrop of ongoing military conflict);

(ii) information about the nature of the inter-operation between military and humanitarian agencies and the role of coordinating agencies, such as the UN, in facilitating such inter-operation;

(iii) information about the types of planning decisions likely to be made in situations similar to those described in the domain scenario (Fig. 1);

(iv) information about the information requirements of key decision makers, e.g. the disposition of military assets, the integrity of transportation routes, the number of people affected by humanitarian incidents, the location of mines and unexploded ordnance, etc.

The knowledge elicited in this phase contributed to the development of the domain ontologies described in Section 4.2, and it also provided some insight into the information needs of key decision-makers, e.g. the need for information about the security situation en route to (or in the vicinity of) a humanitarian incident. This information contributed to some aspects of the design of the AKTiveSA TDS (e.g. it provided constraints on what information was required to support situation awareness and decision-making); however, most design decisions relating to the functionality and user interface of the AKTiveSA TDS were made in conjunction with military stakeholders at a later stage in the project.

During the course of knowledge acquisition, extensive use was made of PCPACK, a dedicated knowledge capture tool marketed by Epistemics. ${ }^{5}$ While this tool proved useful in terms of acquiring and structuring domain knowledge, the native representational format adopted by PCPACK is not (currently) compliant with W3C-endorsed ontology recommendations. This presented something of a problem since we required a migration route to Web Ontology Language (OWL)-based representations without reneging on the distinctive representational and visualization capabilities afforded by PCPACK. Fortunately, some degree of automatic migration can be accomplished since PCPACK-based knowledge repositories are stored in XML format. Nevertheless, the ontology migration initiative proved only partially successful due to the nature of the logical formalisms used by OWL (the problem here is that frame-based knowledge structures cannot easily be converted into the property centric formalisms countenanced by description logic languages). The resulting strategy consisted of a partial translation of primarily taxonomic information to OWL named classes. While a

${ }^{5}$ http://www.epistemics.co.uk/ number of useful tools exist for editing OWL ontologies, most notably the Protégé OWL plugin [19], we have found such environments largely unsuitable in terms of re-presenting knowledge content back to SMEs (who are largely unfamiliar with knowledge engineering techniques). Clearly such experiences highlight the need for tools and methodologies that enable software-assisted knowledge acquisition, while preserving the semantic expressivity of ontology-based formalisms.

\subsubsection{Knowledge modelling}

Due to our need to inter-operate with stakeholder groups, largely unfamiliar with knowledge engineering methods and techniques, we relied on the CommonKADS methodology [17] as a means of developing initial semi-formal knowledge models. Such models reside at a level of formality and semantic resolution that is intermediate between that of the initial knowledge capture products and the subsequent OWL-based representations. The knowledge model component of the CommonKADS model suite provides formalisms for the representation of both static conceptual structures and rule-based contingencies that inhere in the problem domain. On the basis of past experiences, we have found that the level of representational detail afforded by the CommonKADS knowledge model is ideal in terms of enabling communication between stakeholder groups without reneging on the commitment to provide detailed, machine-readable representations of domain-relevant knowledge [20, 21].

\subsubsection{Ontology formalization}

The final phase of the knowledge engineering life-cycle entailed the development of a number of formal ontologies to represent distinct aspects of the problem domain. Due to the desired support for a variety of knowledge-based processes within AKTiveSA, we required the use of a suitably rich and expressive medium for knowledge representation. Recently, attempts to provide a set of representational formalisms for the communication of ontological structures within the framework of the Semantic Web have coalesced around the OWL [18, 22, 23]. We elected to use this language as the representational medium for our ontology engineering activities, in part due to its endorsement by the W3C, its close alignment with Resource Description Framework (RDF) and its level of semantic expressivity, which supports a variety of types of automated reasoning, e.g. subsumption reasoning. The actual ontologies were developed using the Protégé ontology editor with the OWL plugin [24]. The CommonKADS and PCPACK knowledge models were used as the basis for ontology development, and, as described above, some degree of automatic migration was accomplished in the case of PCPACK models.

\subsection{Domain ontologies}

The knowledge infrastructure of the current problem domain transcends a wide variety of domain areas, e.g. meteorology,

The Computer Journal, Vol. 50 No. 6, 2007 
geography, transportation, military conflict, humanitarian relief, etc., and this is reflected in the scope (and scale) of the ontologies developed for the AKTiveSA initiative. These ontologies target the following knowledge areas:

(i) Geography: This ontology deals with all the geographical aspects of the problem domain. It encompasses a wide variety of conceptualizations including terrain features, transport routes, rivers, shorelines, terrain elevation data, etc.

(ii) Transportation: This ontology covers all aspects of transportation in the problem domain. This overlaps, to some extent, with the geography ontology in the sense that transportation routes, e.g. airways and roads, may also be considered elements of the geographical (geo-spatial) domain.

(iii) Meteorology: This ontology deals with all aspects of the climate and weather. The meteorology ontology is important in enabling the system to interpret and utilize information derived from local weather reports and forecasts as well as long term data about regional rainfall, snowfall, seasonal temperature, etc.

(iv) Humanitarian aid: This ontology covers information of relevance to humanitarian operations, i.e. humanitarian hazards (e.g. floods), humanitarian organizations, humanitarian aid programs, humanitarian aid workers, etc.

(v) Military: This ontology includes relevant conceptualisations in the military domain, including tactical operational areas and zones, military platforms, intelligence information, weapons, etc.

(vi) Equipment: This ontology details the various equipment items that may be used in the course of both military and humanitarian operations. It has substantial overlaps with the content of both humanitarian aid and military ontologies.

(vii) Organizations: The organization ontology provides an ontological characterization of various organizations. It includes military organizations, (e.g. the North Atlantic Treaty Organization - NATO), research and monitoring organizations, (e.g. the National Oceanic and Atmospheric Administration - NOAA), religious organizations and sects, and terrorist organizations. Humanitarian organizations are detailed in the humanitarian aid ontology, which imports the constructs defined in the organization ontology.

(viii) Weapons: The weapons ontology is an extension of the military ontology and deals with aspects of weapons systems, including typology and operational status.

\subsection{Knowledge web}

The results of the knowledge engineering initiative were presented in the form of a knowledge web (Fig. 2). This provided a web-based medium for browsing both domain ontologies

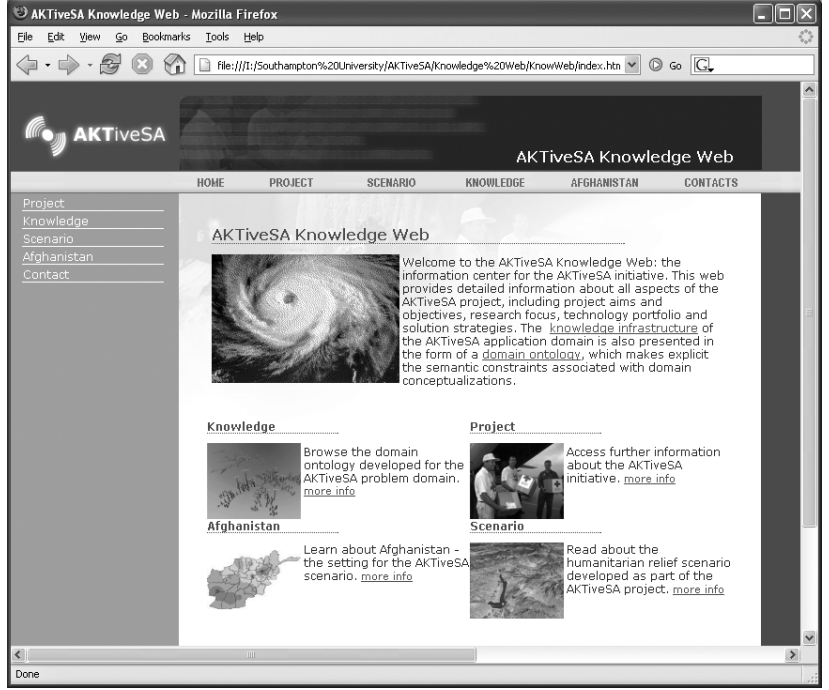

FIGURE 2. AKTiveSA knowledge web.

and knowledge sources. The knowledge web was used by military SMEs as part of the knowledge validation process.

\section{TECHNICAL DEMONSTRATOR SYSTEM}

To showcase the role played by semantically enriched representations in supporting information integration and situation awareness, we developed the AKTiveSA TDS (Fig. 3). The AKTiveSA TDS comprises a suite of applications and technology components, including a knowledge repository, military symbology browser, Semantic Web Application Programmatic Interface, and scenario generation utility. These components have been described in some detail elsewhere [9] and we do not attempt to describe them further here. This section is devoted to a specific component of the AKTiveSA technology portfolio, namely the AKTiveSA TDS Client, a Windows desktop application that allows users to interact with semantically enriched information content via a graphically rich user interface. Subsequent sections describe various features of this application.

\subsection{Earth Browser}

The AKTiveSA TDS Client is built around a NASA component, called Worldwind. ${ }^{6}$ This control co-opts both photorealistic satellite imagery with digital terrain elevation data to provide, what is in effect, a 3-D model of the Earth's surface. The adapted WorldWind component is called the Earth Browser in the context of the AKTiveSA TDS Client (Fig. 4). It allows a user to navigate and visualize any area

\footnotetext{
${ }^{6}$ http://worldwind.arc.nasa.gov/
} 

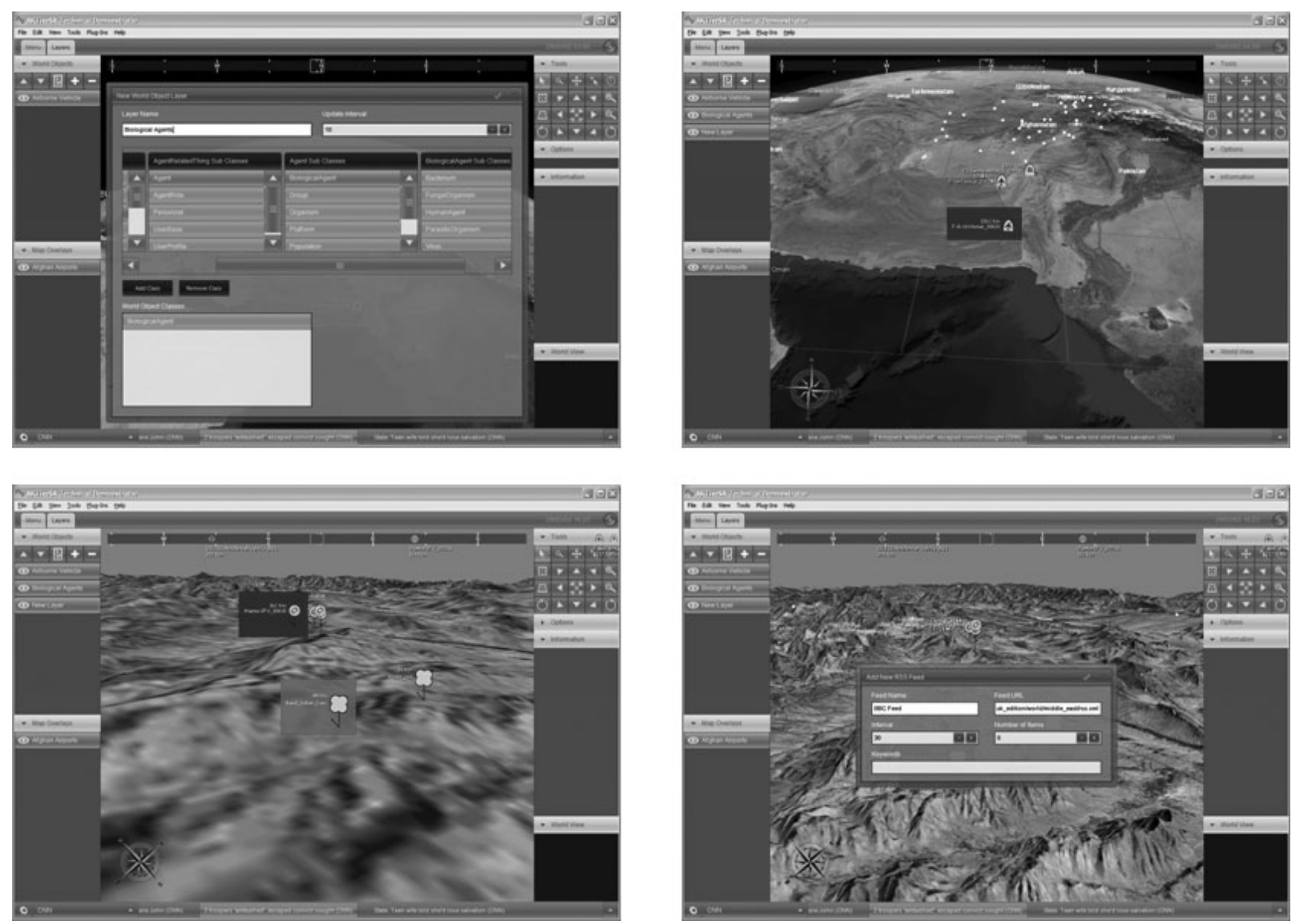

FIGURE 3. AKTiveSA TDS.

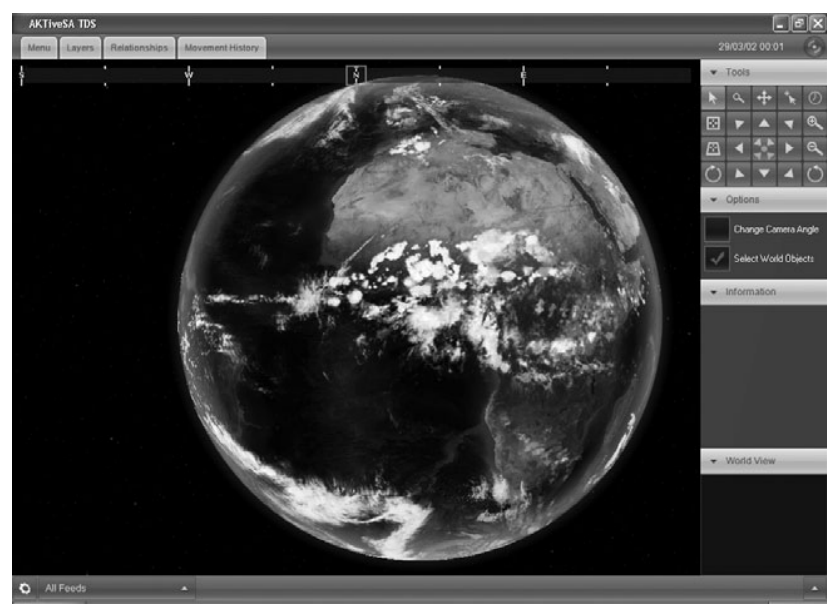

FIGURE 4. Earth Browser.

of the Earth's surface using a variety of navigation controls. The user has complete control of the camera view angle and zoom level using a navigation control palette; similar effects can be accomplished using mouse and keyboard input.

\subsection{Semantic filters}

Semantic filters are used to define selective visualizations of the operational environment; they dictate what types of entities will be displayed in the Earth Browser. A semantic filter could for example be used to selectively display objects of a particular type, or objects with particular characteristics. Ultimately, all semantic filters are implemented as SPARQL queries that periodically execute against the back-end AKTiveSA knowledge repository. The SPARQL query corresponding to each filter is defined using a special interface component (Fig. 5), which allows a user to browse the taxonomic hierarchy of the ontology using a display mechanism inspired by a navigational technology, called mSpace [25]. The user can select multiple classes from the taxonomic hierarchy using this interface component and then specify that these classes be used as part of the semantic filter. The user can also control the frequency with which the query, associated with the filter, is invoked by specifying a time interval for information updates. Once defined, semantic filters appear as toggle buttons in a stack control adjacent to the Earth Browser. These can be toggled on or off by the user to create

The Computer Journal, Vol. 50 No. 6, 2007 


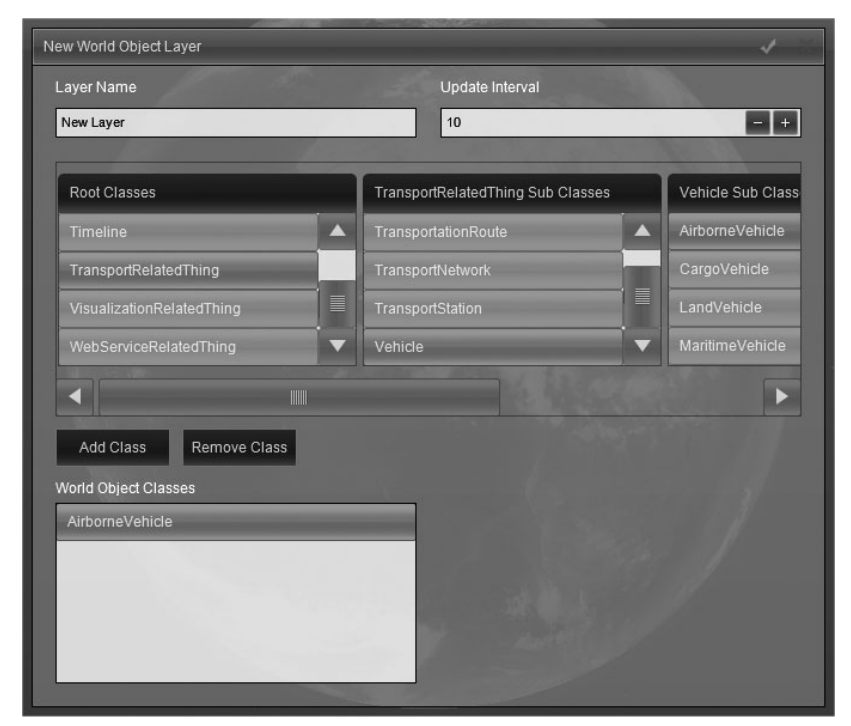

FIGURE 5. Semantic filter component.

complex filtering criteria that juxtapose particular types of information content.

At the present time, the user is restricted to using the concept hierarchy to define semantic filters. An extension of this approach would allow the user to define more complex types of filter, e.g. earthquakes that exceed a certain magnitude, or refugee convoys that are within a certain radius of a particular settlement. Unfortunately, these more complicated filters draw on the full capabilities of the SPARQL query language and this introduces a potential training overhead for the end user. One potential resolution of this difficulty is to allow users to graphically select properties of particular objects; another is to provide graphical query designers that simplify the process of creating complex queries. Both approaches have their drawbacks: graphical query designers may be just as difficult to use as the underlying query language, and the selection of OWL properties presupposes that an ontology imposes constraints on the association between classes and properties. ${ }^{7}$ Future development of the semantic filter capability will, at a minimum, provide a textbased control for SPARQL query construction and we are also considering options for simplified graphical query editors.

\subsection{Compass Bar}

The Compass Bar control (Fig. 6) aids user navigation and spatial orientation within the Earth Browser by representing directional information using compass-like controls. The directional location of objects, such as military platforms, is

\footnotetext{
${ }^{7}$ This is not, in fact, how OWL works. The domains and ranges of OWL properties should not be viewed as constraints to be checked; they are used as axioms in subsumption reasoning processes.
}

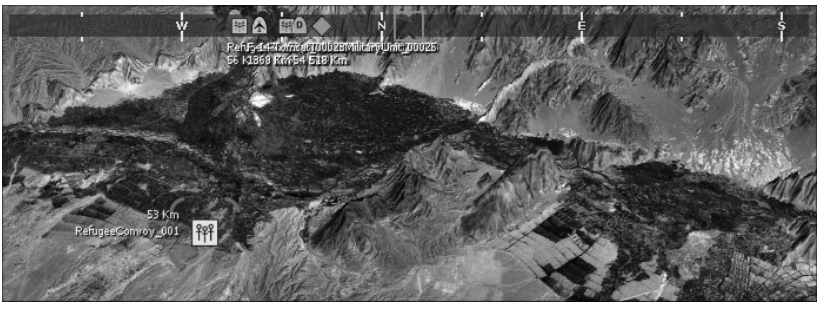

FIGURE 6. Compass Bar control.

represented on the Compass Bar using a small icon, and the user can navigate to objects in the Earth Browser by simply clicking on this icon. The information contents of the Compass Bar are continuously updated as the user navigates around the Earth Browser.

\subsection{Annotation Toolkit}

The Annotation Toolkit consists of a tool palette providing access to tools and utilities that can be used to create new knowledge objects and annotate existing ones. At the present time, the Annotation Toolkit supports the creation of new entities and allows users to annotate geographic regions in the Earth Browser. Future extensions of this capability could provide support for a collaborative form of map annotation similar to that seen in the case of WikiMapia. ${ }^{8}$

\subsection{Property Inspector}

The Property Inspector displays information about an object previously selected in the Earth Browser. The aim of the Property Inspector is to provide quick access to task-relevant information for target objects of interest; however, in the current version of the AKTiveSA TDS Client, the information content of the Property Inspector is not modifiable by the end-user - the control simply provides access to a fixed set of properties for each type of selectable object, e.g. positional and platform status information. In order to adapt the Property Inspector to suit the task-variant information requirements of particular end-users we need to give users control over what information should be displayed in the Property Inspector. The best strategy for this, at the present time, seems to be a semantic query editor or property selection user interface component, similar to that discussed in Section 5.2.

The information contents of the Property Inspector are driven by SPARQL queries that periodically query the back-end knowledge repository for object-specific information. The refresh rate for the Property Inspector is currently fixed, but future implementations will provide a variable refresh rate based on user requirements.

${ }^{8}$ http://wikimapia.org/

The Computer Journal, Vol. 50 No. 6, 2007 


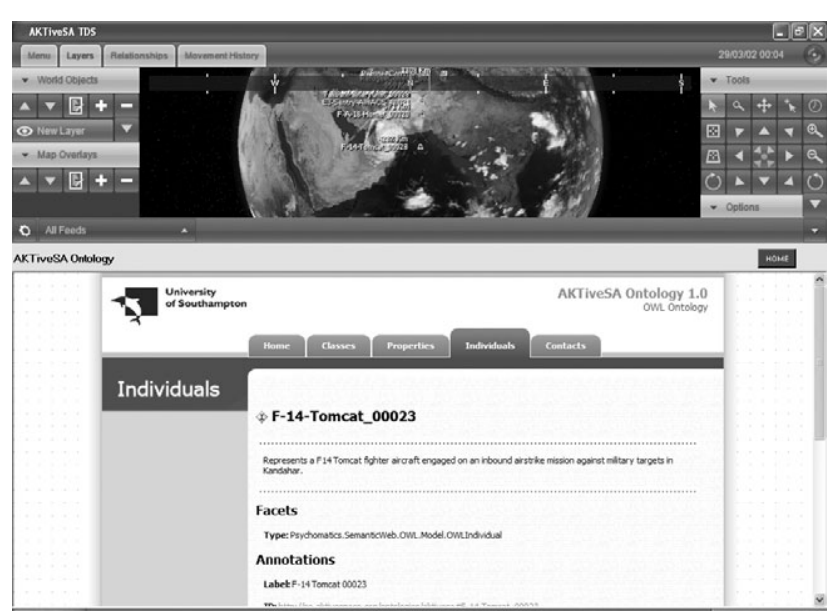

FIGURE 7. Information pane (ontology visualization mode).

\subsection{General Information Pane}

The General Information Pane (Fig. 7) displays general information about scenario objects and resources. It also hosts a copy of the knowledge web described in Section 4.3. The General Information Pane operates in a number of modes:

(i) Resource explorer mode: In this mode the General Information Pane lists any information resources (e.g. images, videos, text files) that are associated with selected objects in the Earth Browser.

(ii) Resource detail mode: In this mode the General Information Pane displays a specific information resource. This could assume the form of a web page, an image, or a video/audio clip. The General Information Pane is based on a web browser control, so it can, in principle, display any type of information resource natively supported by a standard web browser, such as Internet Explorer.

(iii) Ontology visualization mode: In this mode (Fig. 7) the General Information Pane displays the ontological representation of a selected object. All ontology elements within the AKTiveSA ontology are serialized as Hypertext Mark-Up Language (HTML), which, in general, provides a better format for end-user browsing.

(iv) Knowledge web mode: In this mode the General Information Pane displays the knowledge web associated with the AKTiveSA project (Section 4.3).

(v) Information sources list mode: In this mode the General Information Pane displays the information sources for a selected object.

\subsection{RDF Site Summary ticker}

The AKTiveSA TDS Client includes an RDF Site Summary (RSS) ticker control (Fig. 8), which is designed to display information from various user-selected news feeds. The summary

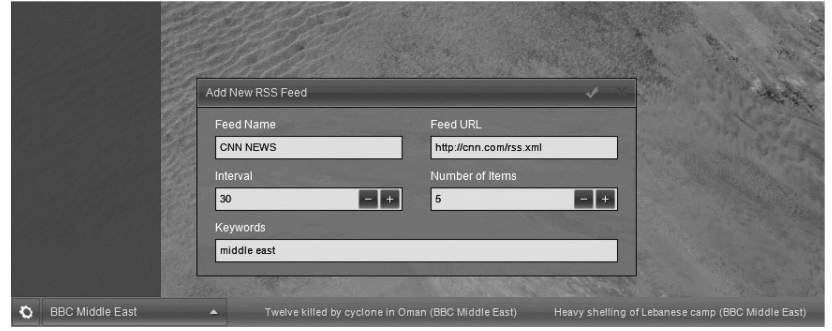

FIGURE 8. RSS ticker control.

for each news item is displayed in a scrolling marquee at the bottom of the user interface and each summary is associated with a 'More Information Button', which, when clicked, will open a dialogue box to display the entire news article. Items that have already been displayed in this way are rendered using a white font, while items that have not previously been displayed are rendered using a red font.

\subsection{Map Overlays}

Map Overlays represent visual information about areas of the terrain as rendered in the Earth Browser. Map Overlays can include vector-based graphics for region-based information, such as population density or language distribution maps. They can also include additional satellite imagery layers. Such overlays provide a means to easily extend the types of information content that can be displayed using the Earth Browser component.

The AKTiveSA TDS includes a limited number of Map Overlay products for the Afghanistan region. These include vector maps for seismicity, population density, language distribution, annual precipitation, natural disaster vulnerability, and land mines/UXO contamination. Such products permit the juxtaposition of scenario-specific information with background Geographic Information System data products for the Afghanistan region.

\subsection{Timeline manipulation}

The AKTiveSA TDS Client provides two means to manipulate the scenario timeline in order to gain a view of both past and future (predicted) states. The Movement Toolbar displays a visible movement trail, which indicates the past and predicted movement of a selected entity (e.g. a military unit) within the Earth Browser display. This visual aid facilitates an understanding of the temporal evolution of the current situation picture, both in terms of past situations and (likely) future situations. A second tool, the Time Gesture Tool, compliments the Movement Toolbar by allowing users to use simple input gestures to alter the current time setting. Horizontal movements from left to right across the screen, using either the mouse (or fingers on a touch-screen interface) advances the temporal

The Computer Journal, Vol. 50 No. 6, 2007 
position forward in time, whereas a gesture from right to left will cause the time setting to regress. Combining horizontal gestures with vertical gestures allows the speed of temporal progress to be manipulated: a movement from the bottom of the screen to the top will increase the speed at which the time changes, allowing for large jumps in temporal position; movements from the top of the screen to the bottom will decrease the speed at which the time changes, allowing for fine-tuning of the temporal setting.

\section{REASONING SUBSYSTEM}

The AKTiveSA TDS technology portfolio originally included a reasoning subsystem to implement situation-relevant inferential processes. Our aim was to support rule execution using an inference engine component and provide decision support to end-users in three specific task areas:

(i) Needs assessment: An assessment of what needs to be done in terms of relief actions and humanitarian aid requirements in order to minimize further harm and alleviate human suffering.

(ii) Relief planning: The actual planning of a relief effort in terms sourcing, delivery and dissemination of aid supplies.

(iii) Future vulnerability assessment: An assessment, or prediction, of the long term implications of the disaster with respect to future humanitarian action.

We successfully developed an inference engine component by adapting the $\mathrm{C}$ Language Integrated Production System (CLIPS $^{9}$ ) expert system shell and we subsequently used the CLIPS programming language to represent domain-specific rules. Unfortunately, however, a number of problems emerged which served to limit the overall utility of the reasoning component. Firstly, the CLIPS inference engine required all triples to be retrieved from the back-end knowledge repository, converted to CLIPS fact assertions and then loaded into the execution environment. This introduced a significant overhead in terms of component initialization, especially in a networked, client-server environment. The problem might have been extenuated by restricting the number of RDF triples to be loaded into the execution environment; however, the various AKTiveSA ontologies were so inter-connected that semantically-insular ontology subcomponents could not be extracted: the need for logical consistency checking and subsumption reasoning necessitated the retrieval of all triples. This may be a general characteristic of semanticallyexpressive knowledge models and, to some extent, it argues in favour of simpler, more modular ontology components.

A second drawback of the reasoning subsystem concerned the performance overhead associated with rule execution. In

\footnotetext{
${ }^{9}$ http://www.ghg.net/clips/CLIPS.html
}

this case, the total time taken for reasoning processes to complete was, generally, not acceptable (some processes took in excess of $12 \mathrm{~h}$ to complete), and, in most cases, the reasoning process did not complete in what could be regarded as an operationally-useful timeframe (i.e. on the order of minutes). One reason for this performance overhead was the number of rule firings made during the reasoning process. The semantic expressivity of ontologies, at least OWL ontologies, supports a large number of rule firings following fact assertion because even a small change to the knowledge infrastructure (i.e. the assertion of a single fact) can have semanticallysignificant implications. Fact assertion may, for example, result in the re-computation of the entire taxonomic hierarchy. Strategies for resolving this problem in future versions of the AKTiveSA TDS include, but are not necessarily limited to the following:

(i) optimization of the inference engine to support faster rule execution;

(ii) intelligent caching of temporary reasoning results;

(iii) progressive minimization of semantic expressivity (i.e. the removal of specific axioms) until a reasonable performance threshold has been attained;

(iv) modularization of ontology components to reduce inter-connectedness; and

(v) more precise control over the firing of specific rule subsets, i.e. only allowing certain rules to fire in a particular reasoning context.

Future research will explore which of these techniques is the most preferable for our purposes in terms of implementing semantically enabled decision support capabilities.

\section{SYSTEM EVALUATION}

Efforts to provide technological support for enhanced situation awareness need to be evaluated in the context of end-user trials. We have not, as yet, conducted such trials with human subjects, but we are currently collaborating with the University of Cardiff to determine how such trials might be undertaken. One of the approaches adopted by the University of Cardiff measures the recall of scenario-relevant information following periods of user interaction with the target system. A number of other measurement techniques for situation awareness have also been proposed, including SAGAT [26, 27], SPAM [28] and SART [29], and we aim to examine and assess the relative suitability of these techniques in subsequent system evaluation studies.

\section{FUTURE WORK}

The capabilities of the AKTiveSA TDS will be refined and extended in future development cycles. This section provides an overview of our current development objectives. 


\subsection{Mobile capabilities}

\subsubsection{Portable devices}

The use of portable devices to access information content is pretty much commonplace in today's society. While the AKTiveSA TDS is optimized for large displays and desktop computing environments, there is no reason why the software could not be ported to mobile devices supporting managed DirectX applications. One aspect of our future work will therefore be to implement mobile versions of the AKTiveSA TDS Client application.

\subsubsection{Mobile information access}

NEC relies on the integrity of communication networks for the purposes of large-scale information dissemination and sharing. However, the increasing emphasis on mobile, ad $h o c$, and wireless networks within military operational contexts presents a number of challenges for NEC. One feature of mobile communications technology, for example, is intermittent network access - the tendency for a mobile device to periodically lose connectivity in areas of poor network coverage. This problem is significant in countries such as Afghanistan, where infrastructure development may be poor or undermined by recent military conflict, and this necessitates a consideration of mechanisms to support operators in occasionally connected operational environments.

One approach to the problem of intermittent network access is provided by data charging techniques [30]. Data charging emphasizes the use of intelligent forward caching mechanisms to pull or push relevant data to mobile devices during periods of high connectivity. This aims to preserve user access to task-relevant information content during subsequent periods of network disconnection. The main challenge with data charging concerns the identification and representation of factors that influence the selection of relevant information. Factors such as problem-solving goals, information requirements, trust ratings (there may be little point in downloading distrusted information), access history (we may be able to infer needs or preferences based on past patterns of information access), the features of the mobile display device (there is no point in downloading information to a display device if the device cannot subsequently display that information to the user) and the profile of the end user may all contribute to adaptive data charging mechanisms [31]. Since such mechanisms are crucial for situation awareness in mobile computing contexts, we intend to explore the notion of adaptive data charging for mobile information access as part of our future development activities.

\subsection{Virtual adviser}

SPARQL queries can be used to represent events or information contingencies of interest to the user, but at present the mechanisms for alerting users to information that satisfies a query, within the context of the AKTiveSA TDS, are somewhat limited: the user is alerted to new information primarily via the RSS ticker control (Section 5.7). Future development initiatives should aim to provide a much richer set of options for user alerts and notifications, including emails, SMS messages, popup dialogues, etc. One additional possibility is to implement a virtual adviser component [32] corresponding to an animated 'talking head'. This component can be used to provide vocalization services for text content and, in most cases, the viscemes, phonemes and emotional tone of the character can be dynamically specified to provide a pseudo-realistic 'conversational' context for information exchange about scenario events and information contingencies. In light of the well-known facilitative effects of multimodal stimuli on dual task performance [33], the exploitation of the auditory modality may support an effective broadening of end-user information processing capacity, especially since the dominant information presentation strategy at present is to exploit the visual modality. The ability to influence the emotional tone of informational messages may be of further value in terms of controlling shifts in attentional focus. Evidence suggests, for example, that emotionally salient stimuli (e.g. angry faces) are more effective in attracting and eliciting attention than neutral or novel stimuli [34].

\subsection{Resource classification}

The web is an unruly place: information content is liable to change, resource locations are frequently modified, existing information all too quickly becomes redundant, and new information is made available at a rate that makes effective manual tracking and monitoring effectively impossible. How are we to deal with such complexity and dynamism? How, in particular, are we to manage the information space such that information consumers are always aware of new information that speaks directly to their problem-solving specific needs, interests and concerns? This is a fundamental problem with large-scale information repositories, such as the WWW, because often the information content of resources is not accessible in a format that can be easily aligned with the kind of conceptual glosses that drive search queries. Current strategies for information search and retrieval, such as keyword searches, are very popular, but they do not avail themselves of the ability to exploit semantic generalizations and they are vulnerable to the vagaries of language style, linguistic variation and transliteration. The result is that important sources of information are lost in the noise of irrelevant links or may not even be returned as part of the search results. One approach to addressing the limitations of keyword searches is to use explicit semantic characterizations of resource content - the strategy adopted by the Semantic Web community. Explicit semantic annotations of information content and information resources are, however, somewhat rare on the WWW and such annotations show no sign of being readily available in the near

The Computer Journal, Vol. 50 No. 6, 2007 


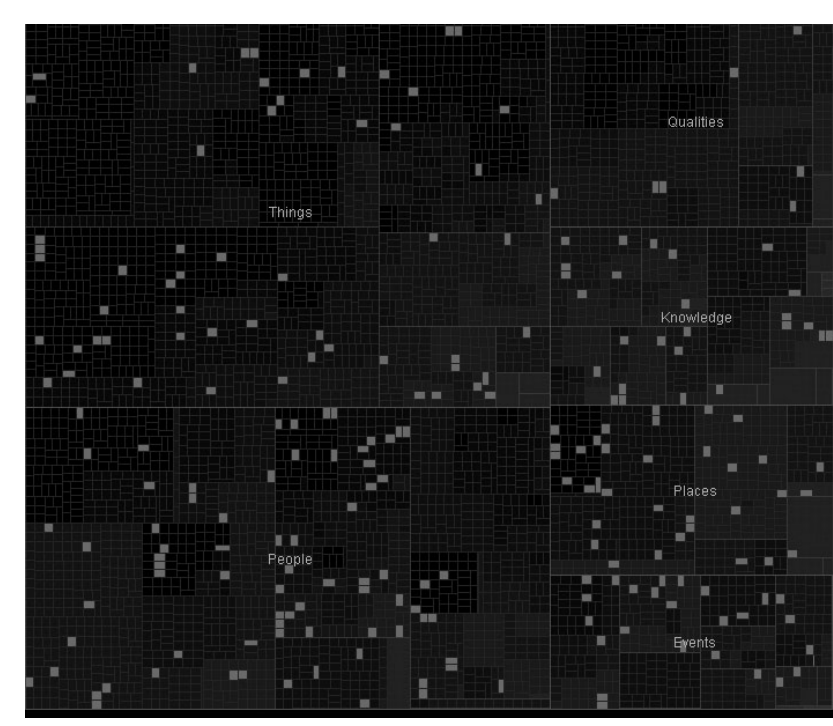

FIGURE 9. Treemap visualization.

future. Given the inadequacy of keyword searches and the paucity of explicit semantic annotations of resource content, alternative strategies for resource discovery and classification are required.

One such strategy involves the use of sophisticated analytic techniques that aim to automatically classify resources and their associated information content with respect to semantically circumscribed topic categories and hierarchies. Machine learning (ML) techniques are particularly good at automatically identifying patterns in data and have recently been applied to text classification problems [35, 36]. The aim of these techniques is to exploit statistical regularities in a training corpus in order to gain a predictive toehold on the classification of novel, previously unseen, resources. A number of studies have demonstrated improvements in MLbased text classification following the integration of background knowledge and semantic constraints. Thie et al. [36], for example, have provided evidence to suggest that improved background knowledge, specifying additional synonym and parent/concept relationships, can be used to improve the correct classification of resources, whilst avoiding a correlative increase in false-positive scores.

Our aim in the context of the DIF DTC Phase II SEMIOTIKS cluster project is to investigate these enhanced capabilities for resource classification using a combination of text analytic techniques and advanced knowledge technologies. Resource classification techniques could provide the basis for sophisticated information management capabilities that organize complex information spaces and facilitate information retrieval. In terms of visualizing such spaces, we have considered the use of interactive treemap displays (Fig. 9), which provide a mechanism for the space-constrained visualization of hierarchical structures [37], e.g. concept hierachies. Treemap displays could be used to allow a user to progressively drill down on more semantically specific areas of a large document space and may provide a useful visualization capability for the outcome of resource classification processes.

\subsection{Content acquisition}

One of the shortcomings of the AKTiveSA TDS is its inability to dynamically extract information content from unstructured textual sources in order to update its knowledge bases with respect to situation-relevant information. Ideally, what is required is a knowledge extraction capability in which relevant domain knowledge can be automatically extracted from source documents. We are currently exploring an ability to augment textual resources with semantic annotations in order to identify the entities contained in the resource document [38, 39]; however, we also intend to explore an additional capability for the extraction of relational information. While the extraction of relational information is significantly more complex than entity recognition, the advantage of the technique is that it provides a much richer substrate for information fusion and knowledge processing, especially in situations where information content is dispersed across multiple source documents. In order to capture relational information we intend to exploit previous work within the Advanced Knowledge Technologies $\left(\mathrm{AKT}^{10}\right)$ initiative, particularly with respect to systems such as Artequakt [40]. Other approaches to content acquisition are also available, e.g. natural language techniques [41] and the scraping of publicly available data from institutional websites [42], and we intend to explore these techniques as part of our future research and development activities.

\subsection{Interaction and visualization}

As part of the continued development of the AKTiveSA TDS, our research into optimal modes of information access, data visualization and user interaction will continue. One relatively recent innovation is the development of a multi-touch screen prototype based on the principle of Frustrated Total Internal Reflection [43]. While single-touch screens are relatively commonplace, multi-touch sensing capabilities enable a user to interact with a display using more than one finger at a time. This can be useful for bi-manual operations, such as using the radial expansion of two digits from a central point to zoom into a map display. Multi-touch capabilities opens up a whole new spectrum of human-computer interaction issues, e.g. how to design affordances that support tactile manipulation of screen elements in a relatively intuitive manner. Multi-touch displays can also support multiple user interaction, which is useful for collaborative working in larger interaction scenarios, such as interactive walls and tabletop displays.

\footnotetext{
${ }^{10}$ http://www.aktors.org/akt/
} 


\section{CONCLUSIONS}

Despite the introduction of new communications technologies and the availability of large-scale information environments, enhancements in situation awareness are not necessarily guaranteed. The rapid advance of information and communications technology has greatly expanded the range and quantity of information available to knowledge workers; however, situation awareness demands more than just the ability to access information content; it also requires an ability to align retrieve, filter and aggregate information with respect to goal-relevant information requirements.

In terms of addressing these concerns we believe the Semantic Web has much to offer, especially in terms of facilitating information exchange, enabling information retrieval (from semantically, lexically and physically disparate information sources), filtering information with respect to dimensions of semantic relevance, and integrating information from multiple semantically heterogeneous source locations.

User interface design is also critical. In the course of user interface design, special attention needs to be devoted to the accessibility of information content, the mechanisms used to alert and notify end-users to specific events, the stimuli used to switch and sustain attentional focus, the representational devices used to convey information and the strategies for manipulating and restructuring information within the context of the user interface display. The AKTiveSA initiative has explored the impact of semantic technologies and advanced user interface design on the potential for enhanced situation awareness within a specific operational context, that of humanitarian and disaster relief operations. The research and development effort has revealed a number of significant challenges in this respect. These include the performance overheads associated with reasoning processes, the difficulty in representing end-user information requirements via semantic query languages, such as SPARQL, and the challenges of real-time knowledge extraction (and integration) from distributed, unstructured textual resources. The resolution of these challenges, and the continued refinement/extension of the AKTiveSA TDS, will be the focus of future development efforts to be carried forward into the second phase of the DIF DTC research programme.

\section{ACKNOWLEDGEMENTS}

We would like to thank Major Glen Burn at the Artillery Targeting Section of the Royal School of Artillery, Larkhill, UK, for his assistance in the knowledge capture and knowledge validation phases of this project. We are also indebted to Lt. Col. Charles Bishop at the Command and Control Development Centre of the Land Warfare Centre, Warminster, UK, for his contribution and feedback during the system design and development phase.
The views and conclusions contained in this document are those of the authors and should not be interpreted as representing the official policies, either expressed or implied, of the UK Ministry of Defence, or the UK Government.

\section{FUNDING}

This work was funded by a grant awarded to the University of Southampton, a part of the Data and Information Fusion Defence Technology Centre (DIF DTC).

\section{REFERENCES}

[1] Berners-Lee, T., Hendler, J. and Lassila, O. (2001) The semantic web. Sci. Am., 284, 34-43.

[2] Seaborne, A. (2004). RDQL - a query language for RDF. http:// www.w3.org/Submission/2004/SUBM-RDQL-20040109/. Wide Web Consortium.

[3] Prud'hommeaux, E. and Seaborne, A. (2005). SPARQL query language for RDF. http://www.w3.org/2001/sw/DataAccess/ rq23/. World Wide Web Consortium.

[4] Endsley, M., Bolte, B. and Jones, D.G. (2003) Designing for Situation Awareness: An Approach to User-Centered Design. Taylor \& Francis.

[5] Endsley, M.R. (2000) Theoretical Underpinnings of Situation Awareness: A Critical Review. In Endsley, M.R. and Garland, D.J. (eds), Situation Awareness Analysis and Measurement. Lawrence Erlbaum Associates.

[6] Waldron, S.M., Morgan, P.L. and Patrick, J. (2006) Designing Integrated Displays for Situation Awareness: Final Report. Technical Report MoD Report DTC/DG(R\&T)/13/25/DIF. School of Psychology, University of Cardiff, Cardiff, Wales, UK.

[7] Kirsch, D. and Maglio, P. (1994) On distinguishing epistemic from pragmatic action. Cogn. Sci., 18, 513-549.

[8] Smart, P.R., Shadbolt, N.R., Carr, L.A. and Shraefel, M.C. (2005). Knowledge-based Information Fusion for Improved Situational Awareness. Proceedings of 8th International Conference on Information Fusion, Philadelphia, USA.

[9] Smart, P.R., Russell, A. and Shadbolt, N.R. (2007) AKTiveSA: Supporting Civil-Military Information Integration in Military Operations Other Than War. Knowledge Systems for Coalition Operations (KSCO), Massachusetts, USA.

[10] JWP 3-52, 2002 (2002) Humanitarian/Disaster Relief Operations. Technical Report. Joint Doctrine and Concepts Centre, Ministry of Defence, UK.

[11] Inter-Agency Standing Committee (2001) Use of Military or Armed Escorts for Humanitarian Convoys. Technical Report, Inter-Agency Standing Committee (IASC). http:// www.humanitarianinfo.org/iasc/content/products/docs/ MCmilescort.doc.pdf

[12] United States Institute of Peace (2002) Good Practices: Information Sharing in Complex Emergencies. Technical Report. United States Institute of Peace (USIP). http:// www.usip.org/virtualdiplomacy/publications/reports/11.html 
[13] Inter-Agency Standing Committee (2004) Civil-Military Relationship in Complex Emergencies: An IASC Reference Paper. Technical Report. Inter-Agency Standing Committee (IASC). http://www.humanitarianinfo.org/iasc/content/ products/docs/CiMi\%2520IASC\%2520Paper\%252028\% 2520June\%25202004.pdf

[14] Smart, P.R. (2005) Knowledge-Intensive Fusion for Improved Situational Awareness: Band Sultan Dam Failure Scenario. Technical Report DTC/WP150/Scenario. Electronics and Computer Science, University of Southampton, Southampton, England.

[15] Shadbolt, N.R. and Burton, M. (1990) Knowledge Elicitation: a Systematic Approach. In Wilson, J.R. and Corlett, E.N. (eds), Evaluation of Human Work: A Practical Ergonomics Methodology. Taylor and Francis.

[16] Shadbolt, N.R., O'Hara, K. and Crow, L. (1999) The experimental evaluation of knowledge acquisition techniques and methods: history, problems and new directions. Int. J. Hum.-Comput. Stud., 51, 729-755.

[17] Schreiber, G., Akkermans, H., Anjewierden, A., de Hoog, R., Shadbolt, N.R., Van de Velde, W. and Weilinga, B. (2000) Knowledge Engineering and Management: The CommonKADS Methodology. MIT Press.

[18] McGuiness, D.L. and van Harmelen, F. (2004) OWL web ontology language overview. http://www.w3.org/TR/ owl-features/. World Wide Web Consortium.

[19] Holger, K., Ferguson, R.W., Noy, N.F. and Musen, M.A. (2004) The Protege OWL Plugin: An Open Development Environment for Semantic Web Applications. Proceedings of 3rd International Semantic Web Conference - ISWC, Hiroshima, Japan.

[20] Smart, P.R. (2001) Development of a Tactical Decision Support System for FOAEW: AEW Barrier Positioning. Technical Report. Epistemics, Nottingham, England.

[21] Smart, P.R. (2002) Development of a Tactical Decision Support System for FOAEW: Mission Planning. Technical Report. Epistemics, Nottingham, England.

[22] Antoniou, G. and van Harmelen, F. (2003) Web Ontology Language: OWL. In Steffen, S. and Studer, R. (eds), Handbook on Ontologies. Springer Verlag.

[23] W3C semantic web website. http://www.w3.org/2001/sw/.

[24] Protégé ontology editor website. http://protege.stanford.edu/.

[25] Schraefel, M.C., Smith, D.A., Owens, A., Russell, A., Harris, C. and Wilson, M.L. (2005) The Evolving mSpace Platform: Leveraging the Semantic Web on the Trail of the Memex. Proceedings of Hypertext, Salzburg, Austria.

[26] Endsley, M. (1988) Design and Evaluation for Situation Awareness Enhancement. Human Factors Society 32nd Annual Meeting, Santa Monica, California, 97-101. Human Factors Society.

[27] Endsley, M.R. (1995) Measurement of situation awareness in dynamic systems. Hum. Factors, 37, 65-84.

[28] Durso, F.T., Hackworth, C.A., Truitt, T.R., Crutchfield, J., Nikolic, D. and Manning, C.A. (1998) Situation awareness as a predictor of performance for en route air traffic controllers. Air Traffic Control Q., 6, 1-20.
[29] Taylor, R.M. (1990) Situational Awareness Rating Technique (SART): The Development of a Tool for Aircrew Systems Design. Situational Awareness in Aerospace Operations (AGARD-CP-478). NATO-AGARD, Neuilly Sur Seine, France.

[30] Cherniack, M., Franklin, M.J. and Zdonik, S. (2001) Expressing user profiles for data recharging. IEEE Pers. Commun. 8, 32-38.

[31] Bahrami, A., Wang, C., Yuan, J. and Hunt, A. (2006) The Workflow based Architecture for Mobile Information Access in Occasionally Connected Computing. Proceedings of IEEE International Conference on Services Computing (SCC'06), Chicago, USA, 406-413.

[32] Taplin, P., Fox, G., Coleman, M., Wark, S. and Lambert, D. (2001) Situation Awareness Using a Virtual Adviser. Proceedings of Talking Head Technology Workshop (OZCHI 2001), Perth, Australia.

[33] Treisman, A.M. and Davies, A. (1973) Divided Attention to Ear and Eye. In Kornblum, S. (ed.), Attention and Performance. Academic Press.

[34] Fenske, M.J. and Eastwood, J.D. (2003) Modulation of focused attention by faces expressing emotion: Evidence from flanker tasks. Emotion, 3, 327-343.

[35] Sebastiani, F. (2005) Text Categorization. In Zanasi, A. (ed.), Text Mining and its Applications. WIT Press.

[36] Thie, C., Lock, Z., Smith, D., Cribb, E., Ford, A. and Rankin, A. (2005) Machine Learning-based Text Analysis for Intelligence Collation. Proceedings of 8th International Conference on Information Fusion, Philadelphia, USA.

[37] Bederson, B.B., Shneiderman, B. and Wattenberg, M. (2002) Ordered and quantum treemaps: Making effective use of 2D space to display hierarchies. ACM Trans. Graph., 21, 833-854.

[38] Ciravegna, F. and Wilks, Y. (2003) Designing Adaptive Information Extraction for the Semantic Web in Amilcare. In Handschuh, S. and Staab, S. (eds), Annotation for the Semantic Web. IOS Press.

[39] Vargas-Vera, M., Motta, E., Domingue, J., Lanzoni, M., Stutt, A. and Ciravegna, F. (2002) MnM: Ontology Driven Semi-automatic or Automatic Support for Semantic Markup. Proceedings of 13th International Conference on Knowledge Engineering and Knowledge Management, Siguenza, Spain. Springer Verlag.

[40] Alani, H., Kim, S., Millard, D.E., Weal, M.J., Hall, W., Lewis, P. and Shadbolt, N.R. (2003) Automatic ontology based knowledge extraction from web documents. IEEE Intell. Syst., 18, 14-21.

[41] Ciravegna, F., Chapman, S., Dingli, A. and Wilks, Y. (2004) Learning to Harvest Information for the Semantic Web. In Bussler, C., Davies, J., Fensel, D. and Studer, R. (eds), The Semantic Web: Research and Applications (First European Web Symposium, ESWS 2004). Springer Verlag.

[42] Leonard, T. and Glaser, H. (2001) Large-scale Acquisition and Maintenance from the Web without Source Access. Proceedings of 4th Workshop on Knowledge Markup and Semantic Annotation, 97-101. ACM Press.

[43] Han, J.Y. (2005) Low-cost Multi-touch Sensing Through Frustrated Total Internal Reflection. Proceedings of 18th Annual ACM Symposium on User Interface Software and Technology, Seattle, Washington, USA, pp. 115-118. ACM Press. 